\title{
A Simulation of Reflected ADS-B Signals over the North Atlantic for a Spaceborne Receiver
}

\author{
Richard Van Der Pryt, Ron Vincent \\ Department of Physics, Royal Military College of Canada, Kingston, Canada \\ Email: Ron.Vincent@rmc.ca
}

Received 30 December 2015; accepted 22 February 2016; published 25 February 2016

Copyright (C) 2016 by authors and Scientific Research Publishing Inc.

This work is licensed under the Creative Commons Attribution International License (CC BY).

http://creativecommons.org/licenses/by/4.0/

(c) (i) Open Access

\begin{abstract}
Automatic Dependent Surveillance-Broadcast (ADS-B) is an air traffic surveillance technology in which aircraft broadcast position, identification and status an average of 6.2 times per second on $1090 \mathrm{MHz}$. The Royal Military College of Canada has developed an ADS-B receiver that is scheduled to fly as a technology demonstrator on the Canadian Advanced Nanospace eXperiment-7 (CanX-7) nanosatellite. A signal propagation model was developed to determine the power level and number of signals that will be detected by CanX-7. Since the ADS-B messages are alternately transmitted from upper and lower antennas, both the direct and reflected signals were considered. A simulation using the model was run over the North Atlantic with aircraft data supplied by air traffic services and a satellite altitude of $800 \mathrm{~km}$. Power at the receiver for reflected ADS-B signals ranged from -109.5 to $-118 \mathrm{dBm}$ depending on aircraft-satellite geometry and was approximately $18 \mathrm{dBm}$ less than the direct path signal strength. With a sensitivity of $-103 \mathrm{dBm}$, the CanX-7 ADS-B receiver should detect virtually all of the direct path signals while reflected signals are below the detection threshold. Although the reflected signals should not be a factor for the CanX-7 mission, they could be a consideration for a large operational satellite with a more sensitive receiver. The reception of both direct and reflected ADS-B signals from multiple aircraft could lead to signal collisions and subsequent loss of aircraft tracking information, particularly in coastal regions where there are additional sources of the $1090 \mathrm{MHz}$ signal.
\end{abstract}

\section{Keywords}

ADS-B, Satellite, Air Traffic Control, Ocean, Scattering

\section{Introduction}

Automatic Dependent Surveillance-Broadcast (ADS-B) is an air traffic surveillance technology in which aircraft routinely transmit aircraft identification, position, velocity and status during flight. Commercial air carriers use 
$1090 \mathrm{MHz}$ to broadcast the 120-bit message at random periods between 0.4 and 0.6 seconds to prevent aircraft from having synchronized transmissions. The ADS-B signal alternates between top- and bottom-mounted quarter-wave monopole antennas, with transmitter power between $75 \mathrm{~W}$ and $500 \mathrm{~W}$ depending on the aircraft category [1]. The transmissions can be received by other aircraft, or by ground stations for relay to Air Traffic Services (ATS) to provide timely and more accurate surveillance information than conventional surveillance radars. In recent years ADS-B has come into use in several regions to provide air traffic information where radar coverage is not available. Canada's ATS provider, NAV CANADA, has implemented ADS-B surveillance in the Hudson Bay corridor and Labrador regions, while airspace authorities in Europe, the United States, and Australia, have plans to implement ADS-B.

ADS-B coverage is currently limited by the placement of ground stations that cannot be installed in midocean and are difficult to maintain in Polar Regions. An orbital ADS-B system has the potential to provide precise aircraft surveillance in remote and oceanic airspace, which would transform existing traffic procedures that use standardized routes and large inter-aircraft spacing to provide separation. An operational spaceborne ADS-B system would reduce aircraft spacing requirements in oceanic regions leading to more efficient routes and subsequent reductions in fuel consumption. These efforts are topical with the announcement that ADS-B receivers are planned as secondary payloads on the Iridium Next constellation [2], a proposed 66-satellite low Earth orbit (LEO) constellation scheduled to be deployed by late 2017.

The Royal Military College of Canada (RMCC) has been involved in space based ADS-B research since 2009, conducting successful high altitude balloon missions and publishing research in the field [3]-[7]. As a result of this work, RMCC developed an ADS-B receiver that is scheduled to fly as a technology demonstrator on the Canadian Advanced Nanospace eXperiment-7 (CanX-7) nanosatellite. Projected for launch in 2016, the ADS-B payload will collect data over the North Atlantic in the Shanwick and Gander Oceanic Control Areas (OCAs) before a de-orbiting payload is deployed. A signal propagation model was created to determine the strength of direct path ADS-B signals arriving at the CanX-7 receiver [7]. Since half of the ADS-B signals are transmitted toward the Earth, transmissions reflected from the ocean surface were also considered since they could potentially double the signal density, leading to signal collisions and subsequent loss of aircraft tracking information. The reflection model takes into account neutral atmosphere and ionospheric effects, aircraft-satellite geometry, sea surface scattering and antenna radiation patterns. The model was used to run a simulation using aircraft data supplied by NAV CANADA and placing the satellite in LEO.

This paper describes the method and results for ADS-B signals reflected from the ocean surface. Section 2 describes the simulation setup; Section 3 focuses on sea surface scattering; Section 4 discusses the simulation results and Section 5 contains conclusions.

\section{Simulation Setup}

The signal propagation model used for the simulation is written in the Python programming language. Aircraft and satellite positions, environmental data, and antenna radiation patterns are input to determine the power of the reflected ADS-B signals reaching a satellite receiver. The model updates once per second, interpolating both aircraft and satellite position and determining relative geometry between transmitter and receiver. The following is a description of aircraft-satellite geometry, reflected signal power and atmospheric effects. Specific parameters used for the simulation are described in Section 4.

\subsection{Aircraft-Satellite Geometry}

The geometry for ADS-B signals transmitted by aircraft to satellite receiver is shown in Figure 1 for both the direct and reflected paths where the aircraft is located at Point A and the satellite at Point D. The geometry shown is described by Equation (1)

$$
2 G_{1}^{3}-3 G G_{1}^{2}+\left[G^{2}-2 a_{e}\left(h_{1}+h_{2}\right)\right] G_{1}+2 a_{e} h_{1} G=0,
$$

in which $G_{1}$ is the distance from the sub-aircraft point to the specular point, Point B. Once $G_{1}$ is determined, the remaining unknowns are readily calculated. This method uses an effective Earth radius, $a_{e}$, described by Equation (2)

$$
a_{e}=k R_{e} \text {, }
$$




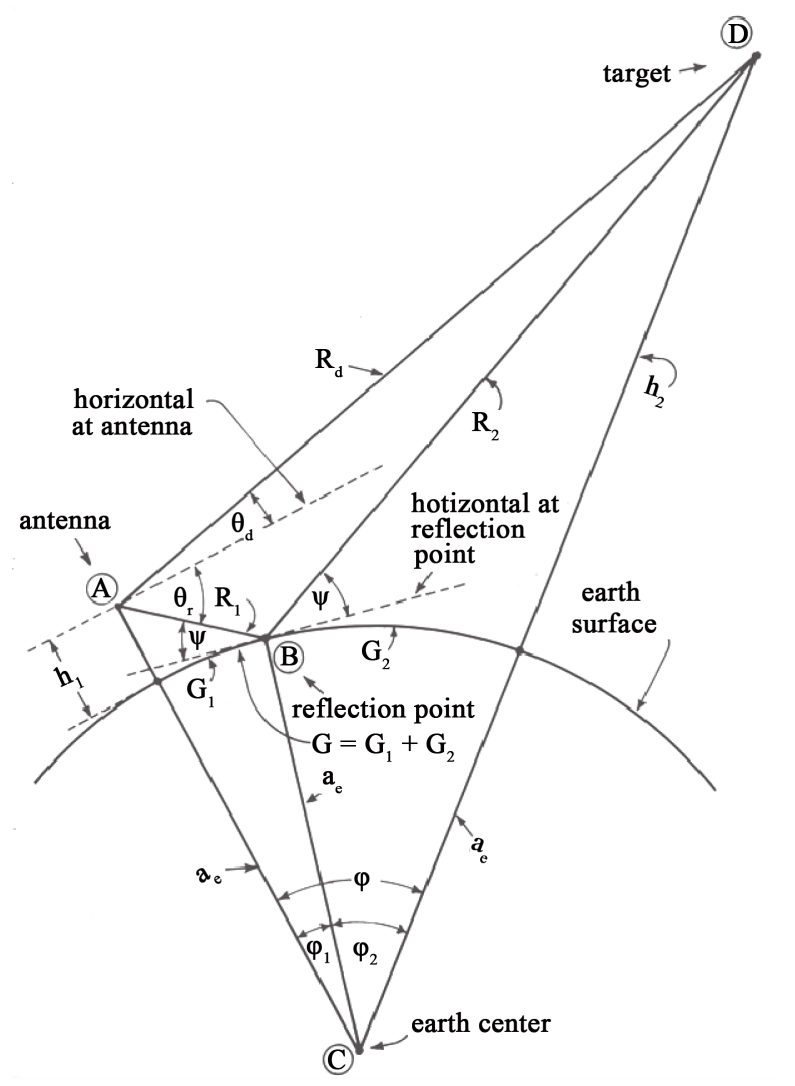

Figure 1. Aircraft-satellite geometry for a spherical-Earth reflection [9].

where $k$ is the radius coefficient and $R_{e}$ is the Earth radius $(6371 \mathrm{~km})$. This factor corrects for atmospheric refraction and permits the assumption of straight ray paths. The value of $k$ varies between 1 for paths perpendicular to the surface to $4 / 3$ for very low grazing angles [8].

Rather than develop a full ray tracing algorithm that would account for the gradient of the refractive index with increasing altitude, a relationship to vary $k$ as a function of elevation angle, $\theta_{d}$, was developed in which

$$
k=\left(1-\frac{\cos \theta_{d}}{4}\right)^{-1} .
$$

For each aircraft-satellite geometry Equation (1) is initially solved for a value of $k=1.0$, then a new $k$ value is calculated from the resultant $\theta_{d}$ using Equation (3). This iterative process is repeated until the solution converges with a tolerance of $\Delta k<1 \times 10^{-7}$. This solution permits $k \approx 4 / 3$ at low grazing angles and reduces to $k \approx 1.0$ at high grazing angles. This approximation was deemed acceptable in light of the greatly reduced computation requirement for the model.

Referring to Figure 1, the method to solve Equation (1) is shown in Equation (4)

$$
G_{1}=\frac{G}{2}-\rho \sin \frac{\xi}{3},
$$

where

$$
\rho=\frac{2}{\sqrt{3}}-\sqrt{a_{e}\left(h_{1}+h_{2}\right)+(G / 2)^{2}}
$$

and

$$
\xi=\sin ^{-1} \frac{2 a_{e} G\left(h_{2}-h_{1}\right)}{\rho^{3}} .
$$


Noting that, $G=G_{1}+G_{2}$ and $\phi=\phi_{1}+\phi_{2}$ from Figure 1, Equation (7) is

$$
\phi_{i}=\frac{G_{i}}{a_{e}},
$$

for $i=(1,2)$. The reflected signal path lengths $\left(R_{1}\right.$ and $\left.R_{2}\right)$ are determined using the law of cosines to triangles $\mathrm{ABC}$ and $\mathrm{BCD}$ as shown in Equation (8)

$$
R_{i}=\sqrt{a_{e}^{2}+\left(a_{e}+h_{i}\right)^{2}-2 a_{e}\left(a_{e}+h_{i}\right) \cos \varphi_{i}} .
$$

The depression angle, $\theta_{r}$, of the reflected path, is found by using the law of cosines on triangle ACD

$$
\theta_{r}=\sin ^{-1} \frac{2 a_{e} h_{1}+h_{1}^{2}-R_{1}^{2}}{2\left(a_{e}+h_{1}\right) R_{1}}
$$

The grazing angle, $\psi$, can be found using triangle $\mathrm{ABC}$

$$
\psi=\sin ^{-1} \frac{2 a_{e} h_{1}+h_{1}^{2}-R_{1}^{2}}{2 a_{e} R_{1}} .
$$

\subsection{Reflected Signal Power}

The bi-static radar equation used to calculate the signal strength observed at the satellite for the sea surface reflection is Equation (11)

$$
P_{\text {reflected }}=\frac{P_{t} G_{t} G_{r} \lambda^{2} D \sigma}{(4 \pi)^{3} R_{1} R_{2} L_{a}},
$$

where $P_{t}$ is the transmitter power, $G_{t}$ and $G_{r}$ are the gains of the transmitting and receiving antennas respectively, $\lambda$ is the wavelength of the electromagnetic wave (0.275 $\mathrm{m}$ for ADS-B), $L_{a}$ represents atmospheric losses, $D$ is divergence and $\sigma$ is the radar cross section (RCS).

Divergence is the weakening of the field strength caused by more rapid spreading caused by reflection from a spherical surface compared to the spreading rate before reflection. In accordance with Blake [9], the divergence factor is described by Equation (12),

$$
D=\left[\frac{a_{e} G \sin \psi \cos \psi}{\left(2 G_{1} G_{2} / \cos \psi+a_{e} G \sin \psi\right)\left(1+h_{1} / a_{e}\right)\left(1+h_{2} / a_{e}\right)}\right]^{-1 / 2} .
$$

In the case of purely specular reflection, $D$ is significant only when the grazing angle is small and can be simplified to Equation (13),

$$
D \approx\left[1+\frac{2 G_{1} G_{2}}{a_{e} G \sin \psi}\right]^{-1 / 2} .
$$

The determination of $\sigma$, which defines the amount of incoming energy reflected from the sea surface boundary, will be discussed in Section 3.

\subsection{Atmospheric Effects}

Potential atmospheric effects on the $1090 \mathrm{MHz}$ signal include Faraday rotation, time delay dispersion, phase dispersion, time delay, ionospheric scintillation and absorption, tropospheric scintillation and absorption and rainfall. Table 1 summarizes atmospheric effects on the ADS-B transmission. Faraday rotation is not a significant factor, particularly for a satellite receiver with a circular polarized antenna. Time delay dispersion and phase angle dispersion do not pose a problem provided that the receiver sample rate is low enough. Time delay is not a factor since the signals arrive at random times and the preamble of ADS-B messages contains synchronization bits. Ionospheric effects are generally of no consequence, although large losses as a result of scintillation may be observed at the geomagnetic equator during equinoctial periods. Small losses are observed in the troposphere as a result of oxygen and water vapor absorption, while rainfall may also incur minor losses to 
Table 1. Atmospheric effects for an ADS-B signal with an elevation angle of $30^{\circ}$ [7].

\begin{tabular}{cc}
\hline Effect & Value \\
\hline Faraday rotation & $12.5^{\circ}$ \\
Time delay & $26 \mathrm{~ns}$ \\
$83 \mathrm{ps}$ \\
Time delay dispersion & $16.3^{\circ}$ \\
Phase dispersion & Up to $20 \mathrm{~dB}$ at the geomagnetic equator at Equinox \\
Ionospheric scintillation & Negligible \\
Ionospheric absorption & $0.114 \mathrm{~dB}$ in rain for multipath case \\
Tropospheric absorption & Negligible for elevation angles $>1^{\circ}$ \\
Tropospheric scintillation & $0.017 \mathrm{~dB}$ in 100 mm $/ \mathrm{hr}$ rain \\
Rainfall & \\
\hline
\end{tabular}

signal power. For reflected signals, neutral atmosphere effects are considered for the downward path of the signal and the subsequent reflected path toward the satellite receiver.

\section{Sea Surface Scattering}

The scattering of the $1090 \mathrm{MHz}$ signal from the sea surface is a key factor in the determination of power received at the satellite. The following subsections describe the calculation of the RCS used in the simulation in terms of scattering theory, reflection coefficients, mean square slope and glistening surface.

\subsection{Scattering Theory}

Efforts to quantify the magnitude of reflected energy from the ocean surface draw on scattering physics, surface wave hydrodynamics and the structure of the marine boundary layer [10]. Increasing interest in this area has been encouraged by numerous datasets collected by microwave sensors on satellite missions such as synthetic aperture radars, scatterometers, and altimeters, or by using reflected satellite microwave transmissions.

The two most commonly used electromagnetic scattering models are the Kirchhoff Approximation (KA) [11] and the Small Perturbation Method (SPM) [12]. The KA method is valid for small slopes of imaginary planes tangential to the surface roughness and long wavelengths of surface roughness relative to the wavelength of the electromagnetic wave. It accurately models quasi-specular scattering but lacks polarization sensitivity. The SPM is valid for small slopes and short wavelengths. It correctly models polarization effects but does not model large features in the surface roughness or specular scattering [10]. Since specular scattering is a significant component of the total energy received in the aircraft-satellite geometry, the SPM method was not selected.

The KA method may be compared with an exact numerical computation of Maxwell's equations using a Multi-Grid Iterative Approach (MGIA) [10]. The basic parameters of this comparison used a $3 \mathrm{GHz}$ signal directed at a one-dimensional random surface that corresponded to a sea surface influenced by a $10 \mathrm{~m} / \mathrm{s}$ wind speed for both vertical and horizontal polarizations at an incidence angle of $30^{\circ}$. The KA method matched the Normalized Radar Cross Section (NRCS), $\sigma^{\circ}$, obtained by the MGIA model for regions bounded by $\pm 25^{\circ}$ around the specular reflection for both polarizations. The KA method underestimated the NRCS for the vertical polarization and overestimated the NRCS for the horizontal polarization in the more diffuse scattering regions greater than $\pm 25^{\circ}$ around the specular reflection. As the model developed in this paper calculates the magnitude of a specularly reflected ADS-B signal in the region where the approximation matched the exact calculation, the KA method was selected as a result of its reduced computational requirements.

\subsection{Radar Cross Section}

The radar equation, Equation (11), used to calculate the magnitude of the ADS-B signal relies on determining the NRCS $\left(\sigma^{\circ}\right)$, for a given surface roughness and then multiplying it by the area that contributes to the reflection to arrive at the RCS $(\sigma)$. Kodis [13] used the stationary-phase approach to describe the scattering from a rough 
surface. His analysis evaluated the physical optical integral from Maxwell's equations before averaging over a rough surface rather than afterwards. Kodis concluded that the average scattered power is proportional to the average number of specular points on the surface times the average curvature of these points, and that the scattering problem is therefore a problem in the statistical geometry of irregular surfaces. Barrick [14] developed this idea further by deriving expressions for the average number of specular points and the Gaussian surface curvature. Since the specular points are separated by many wavelengths, the final expression is the incoherent sum of the individual scattering cross sections. For a surface where the surface heights are Gaussian distributed, the NRCS in the bi-static case is expressed by Equation (14)

$$
\sigma_{\xi \eta}^{o}=\frac{\text { sec }}{m s s} \exp \left(-\frac{\tan ^{2} \beta}{m s s}\right)\left|R_{\xi \eta}(l)\right|^{2},
$$

where $\eta$ and $\xi$ are the scattered and incident polarization states respectively, mss is the mean square of the total slope at a point on a two-dimensionally rough surface, $l$ is the local angle of incidence at the specular point, $\beta$ is the angle between the mean normal to the surface and the local surface normal at the specular point, and $R$ is the refection coefficient. Figure 2 illustrates the geometry described in Equation (14).

\subsubsection{Reflection Coefficient}

ADS-B antennas on aircraft are mandated to operate within $3 \mathrm{~dB}$ of an ideal quarter-wave monopole in the same plane of the aircraft [1]. Specular point theory assumes incident radiation is reflected from a plane tangent to the surface at that point. The reflection coefficients for a vertically polarized incident wave, in terms of the angles in Figure 2 are shown in Equation (15) and Equation (16)

$$
\begin{gathered}
R_{v h}=\sin \varphi_{s} \frac{a_{2} \sin \theta_{s} R_{h}-a_{3} \sin \theta_{i} R_{v}(l)}{4 \sin ^{2} \imath \cos ^{2} \imath} \\
R_{v v}=\frac{\sin \theta_{i} \sin \theta_{s} \sin ^{2} \varphi_{s} R_{h}-a_{2} a_{3} \sin R_{v}(l)}{4 \sin ^{2} \iota \cos ^{2} \imath}
\end{gathered}
$$

where

$$
\begin{aligned}
& a_{2}=\cos \theta_{i} \sin \theta_{s}+\sin \theta_{i} \cos \theta_{s} \cos \varphi_{s} \\
& a_{3}=\sin \theta_{i} \cos \theta_{s}+\cos \theta_{i} \sin \theta_{s} \cos \varphi_{s} .
\end{aligned}
$$

$R_{v h}$ is the reflection coefficient for a vertically polarized incident wave and a horizontally polarized scattered wave, and $R_{v v}$ is the reflection coefficient for a vertically polarized incident wave and a vertically polarized

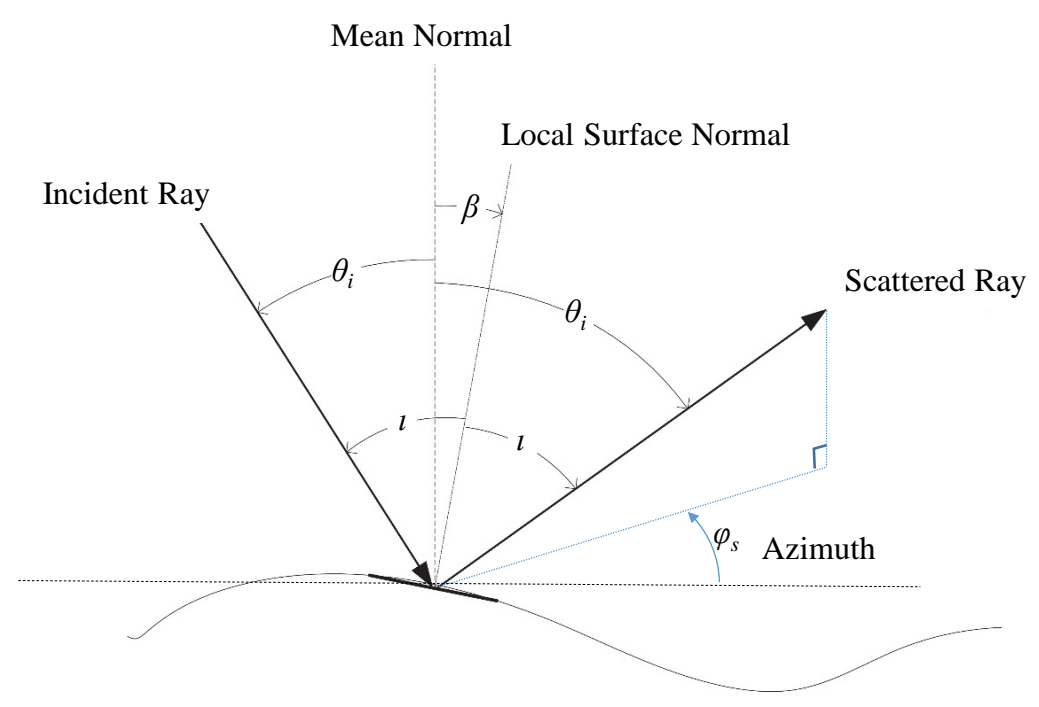

Figure 2. Bi-static radar scattering geometry for a small tangential plane reflecting surface. 
scattered wave. The quantities $R_{v}(l)$ and $R_{h}(l)$, shown in Equation (19) and Equation (20) are the Fresnel reflection coefficients for vertical and horizontally polarized waves [14]

$$
\begin{gathered}
R_{v}(l)=\frac{\varepsilon \cos \imath-\sqrt{\varepsilon-\sin ^{2} \imath}}{\varepsilon \cos \imath+\sqrt{\varepsilon-\sin ^{2} \imath}}, \\
R_{h}(l)=\frac{\cos \imath-\sqrt{\varepsilon-\sin ^{2} \imath}}{\cos \imath+\sqrt{\varepsilon-\sin ^{2} \imath}},
\end{gathered}
$$

where $\varepsilon$ is the complex dielectric constant of seawater. For the simulation, $\varepsilon$ was calculated as a function of sea surface temperature and salinity using a double Debye relaxation law [15].

With the geometry described in Figure 1, where the source and receiver lie in the x-z plane, $\varphi_{s}$ is zero or near zero, which means that $R_{v h}=0$ from Equation (15). With $\varphi_{s}=0$, the reflection coefficient for the simulation is a simplification of Equation (16) and is expressed in Equation (21)

$$
R_{v v}=\frac{-a_{2} a_{3} \sin R_{v}(i)}{4 \sin ^{2} i \cos ^{2} i}
$$

\subsubsection{Mean Square Slope}

Elfouhaily et al. [16] developed a two-dimensional statistical distribution of wind-generated surface waves that combined high and low wavenumber contributions into a unified model. This model corrected the deficiencies of previous full wavenumber models [17]-[19] and exhibited good agreement with a large body of observations [20]-[22]. It has become the de facto standard wave spectra model.

Two main parameters used in oceanographic research to calculate wave spectra are the wind at $10 \mathrm{~m}$ reference height above the sea surface (U10) and the Significant Wave Height (SWH). SWH is defined as the mean wave height from trough to crest of the highest third $\left(\mathrm{H}_{1 / 3}\right)$ of the waves as this is the mathematical value closest to that estimated by trained observers. SWH values are available from oceanographic prediction tools such as the NOAA Wave Watch III model. These parameters alone are not sufficient to characterize wave spectra since distance the wind acts on the surface, or fetch, also has an effect. Wave height increases asymptotically with fetch to a limit where gravitational effects balance wind energy. The Elfouhaily model uses a parameter, known as inverse wave age $\left(\Omega_{\mathrm{c}}\right)$, to describe the effects of fetch. For example, a fully developed sea has a value of $\Omega_{\mathrm{c}}$ close to 0.84 . The omnidirectional elevation spectrum, $S(k)$, is the sum of the low and high wave spectra [16], as shown in Equation (22)

$$
S(k)=k^{-3}\left(B_{l}-B_{h}\right),
$$

where $k$ is the wavenumber, $B$ is the curvature spectrum, and the subscripts $l$ and $h$ indicate the low and high frequencies respectively. Figure 3 shows $S(k)$ over the range of $k=10^{-3}$ to $10^{2}$ for $\mathrm{U}_{10}$ wind speeds between 3 and $21 \mathrm{~m} / \mathrm{s}$. Of note is the relative insensitivity of $S(k)$ to changes in wind speed for wavenumbers between 2 and $10 \mathrm{rad} / \mathrm{m}$.

For isotropic Gaussian distributed slopes, mss can be derived from the sea surface elevation spectrum by integrating over wavenumbers $k$ as shown in Equation (23)

$$
m s S=\int_{0}^{\infty} k^{2} S(k) \mathrm{d} k .
$$

Elfouhaily's model sets the limits of the integral to 0 and $\infty$. The upper limit is problematic as the wave spectra at high wavenumbers does not approach an asymptote as the spectra does at the low wavenumbers. To counter this problem while fitting experimental observations, Zavorotny and Voronovich [23] introduced an upper limit of $k_{*}=2 \pi / 3 \lambda$ as variances of large-scale slopes in a two-scale model are predominantly determined by the low frequency region where $k<k_{*}$. In a 2012 telephone conversation, Dr. Zavorotny modified his equation for $k_{*}$ to include cosine of the incident angle to better fit observations at low grazing angles. The equation to determine mss then becomes Equation (24)

$$
m S S=\int_{0}^{\frac{2 \pi}{3 \lambda} \cos \theta_{i}} k^{2} S(k) \mathrm{d} k .
$$




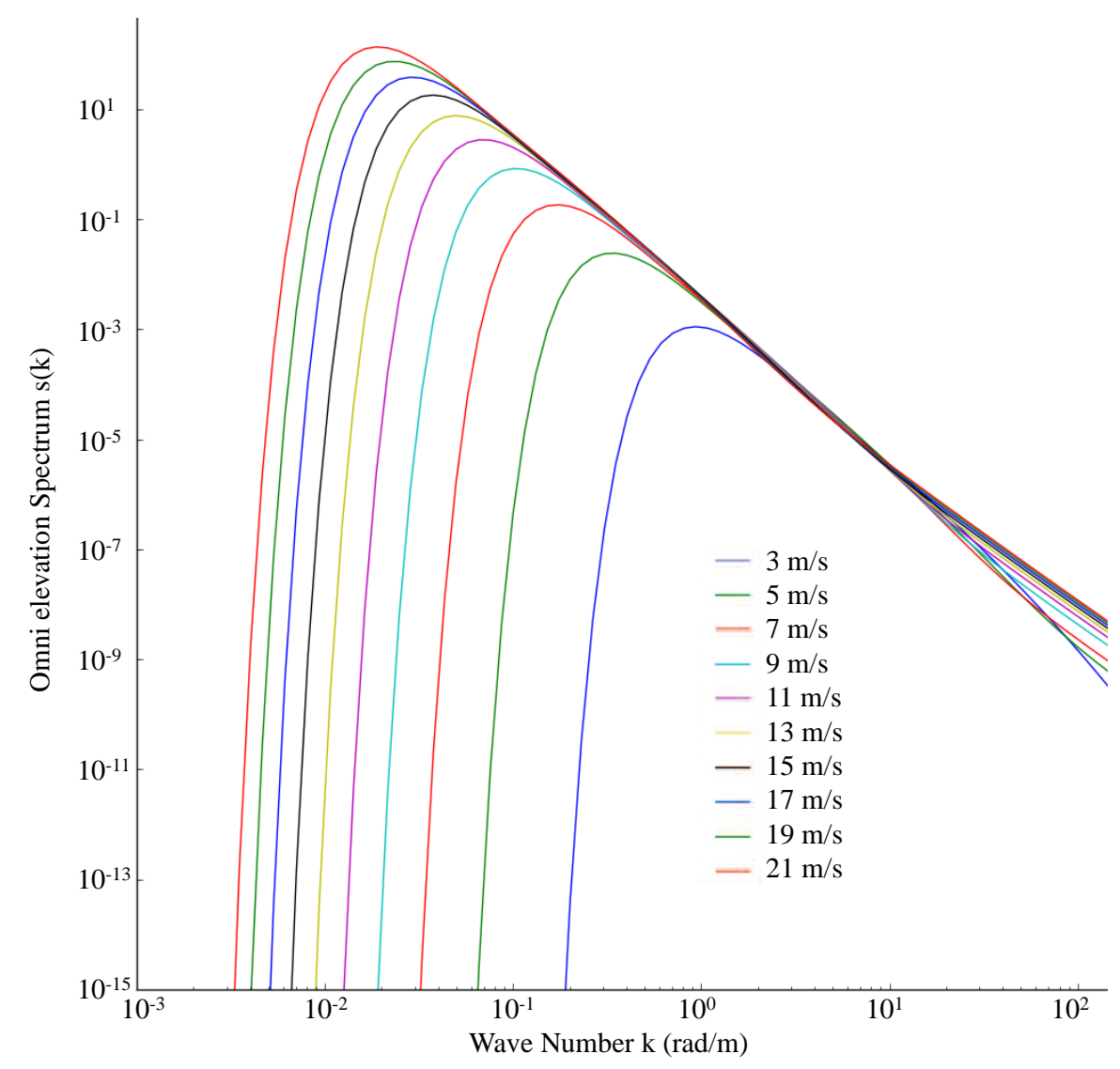

Figure 3. Omnidirectional wave spectra $S(k)$ for U10 wind speeds between 3 and $21 \mathrm{~m} / \mathrm{s}$ for fully developed seas.

\subsubsection{Glistening Surface}

As the calculations to determine the NRCS rely on the contribution of a unit area to the aggregate, it is necessary to calculate the area of the glistening surface (AGS). The term glistening surface defines the portion of the Earth's surface that can contribute to the reflection for a given transmitter/receiver geometry. The model used by Beckmann and Spizzichino [11] assumes the reflecting surface is composed of small tangential planes where $\alpha$ is the distribution of slopes and the maximum slope, $\alpha_{\max } \equiv \beta_{o}$, where $\tan ^{2} \beta_{o}$ represents the mean square slope of the irregularities. This relationship allows the calculation of the maximum surface slope from $S(k)$ as shown in Equation (25)

$$
m s S=\tan ^{2} \beta_{o}=\int_{0}^{\frac{2 \pi}{3 \lambda} \cos \theta_{i}} k^{2} S(k) \mathrm{d} k .
$$

Normalizing by the NRCS at the specular point $(\beta=0)$ yields a step function [11], which is illustrated by Equation (26) and Equation (27)

$$
\begin{aligned}
& G_{0}=\left(\beta, \beta_{o}\right)=1 \text { for } \beta \leq \beta_{o}, \\
& G_{0}=\left(\beta, \beta_{o}\right)=0 \text { for } \beta>\beta_{o} .
\end{aligned}
$$

This function represents the multiplication of the NRCS by the area bounded by $\beta=\beta_{0}$. An easy way to visualize this is to think of a cone with a vertex angle of $\beta_{o}$ coming from the aircraft and centered on the path from the aircraft to the specular point (path AB in Figure 1). The shape of the bounded area is circular at a normal incidence, transforms to roughly elliptical at intermediate angles of incidence, further transforms to a truncated paraboloid and then to a truncated hyperboloid at high incidence angles. A plane tangent to the Earth's surface at 
the specular point was used to determine the area of the glistening surface rather than calculating the area on a sphere. This approach was adopted to reduce computation time for a first-order model, although it tended to slightly underestimate the size of the glistening area at high incidence angles. This area underestimation is acceptable as a spherical surface, having a slightly larger area, would be also be subject to divergence that would reduce the reflected field strength.

As the maximum signal is received when the scattered angle, $\theta_{s}$, is the same as the incident angle $\theta_{i}, \beta$ goes to zero and $i=\theta_{i}$. This simplifies Equation (14) for the VV polarization case to Equation (28)

$$
\sigma=\sigma_{v v}^{o} A G S \text {. }
$$

Determining AGS by the step function described in Equation (26) and Equation (28) results in Equation (29)

$$
\sigma_{v v}^{o}=\frac{\left|R_{v v}\left(\theta_{i}\right)\right|^{2}}{m s s}
$$

This value of $\sigma$ can be directly substituted into the bi-static radar equation shown in Equation (11).

\section{Results}

The simulation presented in this paper is based on a satellite in LEO passing over the north Atlantic Gander and Shanwick OCAs. The scenario was run for altitudes between 400 and $800 \mathrm{~km}$. The receiving antenna is a circularly polarized patch antenna with a half-beam width of $55^{\circ}$, similar to that planned for the CanX-7 mission. This gives a receiver footprint of approximately $2800 \mathrm{~km}$ at $800 \mathrm{~km}$ altitude. Aircraft position and altitude data was obtained from NAV CANADA for a 24-hour period on 29 April 2012 as shown in Figure 4. There is a peak of 218 aircraft at 0300 UTC and another peak of 216 aircraft at 1430 UTC, representing the eastward and westward flow of air traffic respectively.

Aircraft are fitted with antennas on the upper and lower surfaces to achieve spherical coverage. An average of 6.2 ADS-B messages are transmitted per second, alternating between the upper and lower antenna. The aircraft transmitting antenna for the scenario was the S65-5366 made by Sensor Systems, which is used by Boeing and Airbus commercial fleets. Figure 5 shows the antenna radiation pattern for the S65-5366 antenna at $1100 \mathrm{MHz}$. For the simulation it was assumed that the left-hand side of the longitudinal pattern is rotated about the z-axis so that there is no azimuthal dependence.

Figure 6 shows the results of the simulation for a satellite altitude of $800 \mathrm{~km}$, which is the anticipated orbital altitude for CanX-7 and is close to the projected $780 \mathrm{~km}$ altitude of Iridum NEXT. Signal strength for the direct and reflected path of the ADS-B signal are shown from satellite nadir out to approximately $60^{\circ}$. The simulation demonstrated that the reflected ADS-B signal strength is insensitive to wave height, which corresponds to forward scattering results using GPS signals [23]-[27]. Adjusting the mss to account for near surface wind speeds

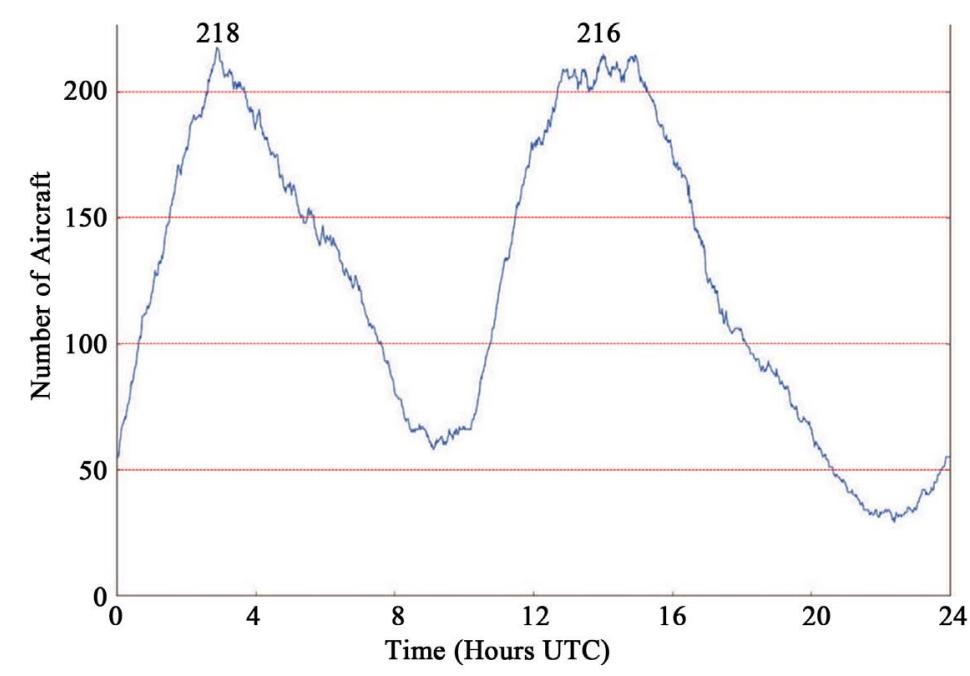

Figure 4. Aircraft traffic in Gander/Shanwick OCAs on 29 Apr 2012. 


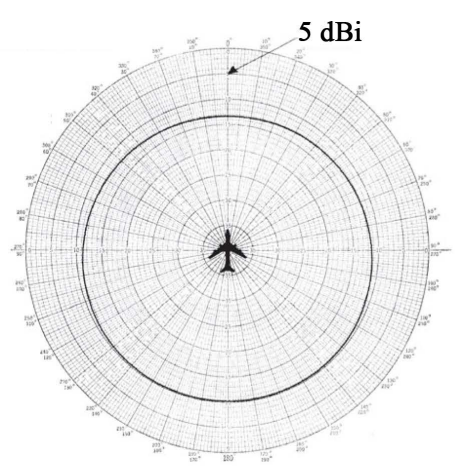

(a)

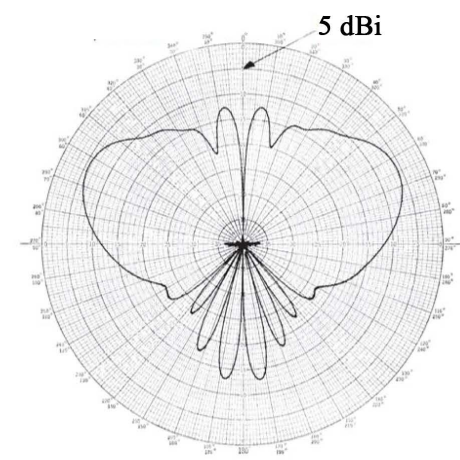

(b)

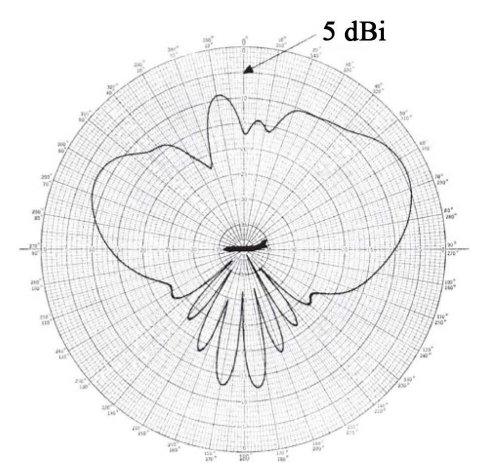

(c)

Figure 5. S65-5366 Antenna Radiation Pattern at 1100MHz for (a) azimuth; (b) longitudinal and (c) lateral directions (Sensor System, Inc.) [7].

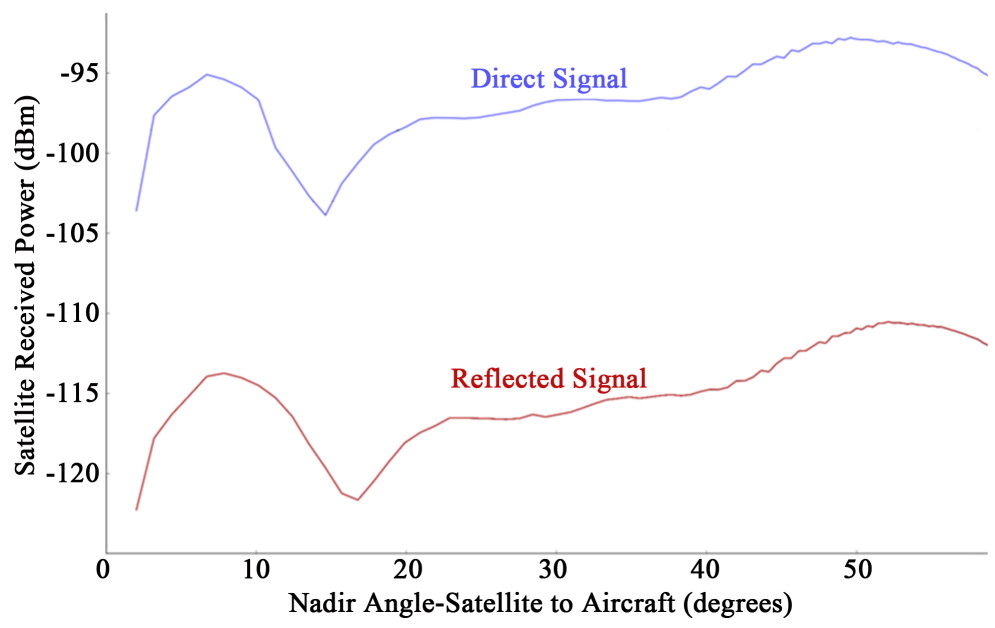

Figure 6. ADS-B reflected signal strength observed at satellite at $800 \mathrm{~km}$ altitude.

from 3 to $21 \mathrm{~m} / \mathrm{s}$ had no effect on the reflected signal strength since the increase in the AGS for rougher seas compensates for the reduced forward scattering per unit area. There is a null directly beneath the satellite and a marked decrease in strength at about $17^{\circ}$ to $19^{\circ}$. The null that occurs when the aircraft is at the satellite nadir is typical of a quarter wave monopole (Figure 5(a)). With an orbital speed of $7.45 \mathrm{~km} / \mathrm{s}$ for an $800 \mathrm{~km}$ orbit, this null configuration would last for a maximum of 10 seconds. The decrease in received power between $17^{\circ}$ and $19^{\circ}$ appears to be related to the lateral radiation pattern of the aircraft antenna (Figure 5(c)). The received power of the reflected signal ranges between -109.5 to $-118 \mathrm{dBm}$ between $2^{\circ}$ and $60^{\circ}$ and is approximately $18 \mathrm{dBm}$ lower than the direct path signal. The details for the simulation of the direct path signal using the model are available in [7]. Simulations run for satellite altitudes of 600 and $400 \mathrm{~km}$ show similar signal strength patterns with and an overall increase in signal strength of 3 and $6 \mathrm{dBm}$ respectively.

With a sensitivity of $-103 \mathrm{dBm}$, the CanX-7 ADS-B receiver should detect virtually all of the direct path signals while reflected signals are below the detection threshold. For an operational constellation such as Iridium NEXT, which is likely to feature ADS-B receivers with greater sensitivity than a nanosatellite, the reflected signals could potentially be detected. The increase in signal density could lead to signal collisions and subsequent loss of aircraft tracking information, particularly near coastal areas where there are other sources of $1090 \mathrm{MHz}$ including Modes A, C, S transponders and Traffic Collision Avoidance System (TCAS).

\section{Conclusions}

The CanX-7 nanosatellite, due for launch in 2016, will host an RMCC payload that will monitor aircraft-generated $1090 \mathrm{MHz}$ ADS-B transmissions over the North Atlantic. In an effort to determine the number and strength 
of signals reaching the receiver, a signal propagation model was created, taking into account neutral atmosphere and ionospheric effects, aircraft-satellite geometry and antenna radiation patterns. A signal propagation model was created to determine the strength of direct path ADS-B signals arriving at the CanX-7 receiver [7]. Since half of the total ADS-B transmissions originate from aircrafts' lower antenna, the reflection of the $1090 \mathrm{MHz}$ signal from the sea surface must also be considered. The signal propagation model was extended to include forward scattering of the ADS-B signal through the determination of aircraft-satellite geometry, antenna radiation pattern and the resulting reflection coefficient and area of the glistening surface.

A simulation using the model was run over the North Atlantic with aircraft data supplied by NAV CANADA at satellite altitudes of $400-800 \mathrm{~km}$. The simulation examined the power received at the satellite of reflected ADS-B signals for wind speeds of 3 to $21 \mathrm{~m} / \mathrm{s}$. The key findings of the simulation are as follows.

1) At an altitude of $800 \mathrm{~km}$ the received power of the reflected signal ranges between -109.5 to $-118 \mathrm{dBm}$ between $2^{\circ}$ and $60^{\circ}$ from satellite nadir and is approximately $18 \mathrm{dBm}$ lower than the direct path signal.

2) There is no relationship between the wave height, which is related to near surface wind speed, and the received signal.

3) There is a null when the aircraft is at satellite nadir as a result of the quarter-wave monopole antenna radiation pattern.

4) For every $100 \mathrm{~km}$ reduction in satellite altitude below $800 \mathrm{~km}$ there is approximately a $1.5 \mathrm{dBm}$ increase in signal strength for both the direct and reflected ADS-B signal.

The simulation indicates that the CanX-7 ADS-B patch antenna, with a sensitivity of $-103 \mathrm{dBm}$, will not detect the reflected signals at the anticipated altitude of $800 \mathrm{~km}$. However, the scattered signals could be a consideration for a larger satellite with a more sensitive receiver. In coastal regions in which there are increased sources of $1090 \mathrm{MHz}$, the cumulative effect of direct and reflected ADS-B signals could lead to signal collisions and loss of aircraft tracking information.

\section{References}

[1] RTCA DO-260B (2009) Minimum Operational Performance Standards for $1090 \mathrm{MHz}$ Extended Squitter Automatic Dependent Surveillance-Broadcast (ADS-B) and Traffic Information Services-Broadcast (TIS-B). Radio Technical Commission for Aeronautics.

[2] Gupta, O.P. (2011) Global Augmentation of ADS-B Using Iridium NEXT Hosted Payloads. Proceedings of the Integrated Communications, Navigation and Surveillance Conference (ICNS), Herndon, 10-12 May 2011, 1-15. http://dx.doi.org/10.1109/icnsurv.2011.5935399

[3] Francis, R., Vincent, R., Noël, J.M., Tremblay, P., Desjardins, D., Cushley, A. and Wallace, M. (2011) The Flying Laboratory for the Observation of ADS-B Signals. International Journal of Navigation and Observation, 2011, Article ID: 973656. http://dx.doi.org/10.1155/2011/973656

[4] Francis, R., Noël, J. and Vincent, R. (2011) Orbital Monitoring of Automatic Dependent Surveillance—Broadcast (ADS-B) Signals for Improved Air Traffic Surveillance in Remote and Oceanic Airspace. Proceedings of the 62nd International Astronautical Congress.

[5] Cushley, A. and Noël, J.M. (2014) Ionospheric Tomography Using ADS-B Signals. Radio Science, 49, 549-563. http://dx.doi.org/10.1002/2013RS005354

[6] Van Der Pryt, R. and Vincent, R. (2015) A Simulation of Signal Collisions over the North Atlantic for a Spaceborne ADS-B Using Aloha Protocol. Positioning, 6, 23-31. http://dx.doi.org/10.4236/pos.2015.63003

[7] Van Der Pryt, R. and Vincent, R. (2015) A Simulation of the Reception of Automatic Dependent SurveillanceBroadcast (ADS-B) Signals in Low Earth Orbit. International Journal of Navigation and Observation, 2015, Article ID: 567604. http://dx.doi.org/10.1155/2015/567604

[8] ITU-R P.834 (2007) Effects of Tropospheric Refraction on Radiowave Propagation. International Telecommunication Union-Radiocommunication Sector, Study Group 3.

[9] Blake, L.V. (1986) Radar Range-Performance Analysis. Artech House, 443 p.

[10] Jackson, C.R. and Apel, J.R. (2004) Synthetic Aperature Radar Marine User's Manual. U.S. Department of Commerce, $464 \mathrm{p}$.

[11] Beckmann, P. and Spizzichino, A. (1987) The Scattering of Electromagnetic Waves from Rough Surfaces (Artech House Radar Library). Artech House, Dedham, 512 p.

[12] Ulaby, F.T., Moore, R.K. and Fung, A.K. (1982) Microwave Remote Sensing, Active and Passive: Volume II, Radar Remote Sensing and Surface Scattering and Emission Theory. Longman Higher Education, 634 p. 
[13] Kodis, R. (1966) A Note on the Theory of Scattering from an Irregular Surface. IEEE Transactions on Antennas and Propagation, 14, 77-82. http://dx.doi.org/10.1109/TAP.1966.1138626

[14] Barrick, D. (1968) Rough Surface Scattering Based on the Specular Point Theory. IEEE Transactions on Antennas and Propagation, 16, 449-454. http://dx.doi.org/10.1109/TAP.1968.1139220

[15] Meissner, T. and Wentz, F.J. (2004) The Complex Dielectric Constant of Pure and Sea Water from Microwave Satellite Observations. IEEE Transactions on Geoscience and Remote Sensing, 42, 1836-1849. http://dx.doi.org/10.1109/TGRS.2004.831888

[16] Elfouhaily, T., Chapron, B., Katsaros, K. and Vandemark, D. (1997) A Unified Directional Spectrum for Long and Short Wind-Driven Waves. Journal of Geophysical Research, 102, 15781-15796. http://dx.doi.org/10.1029/97jc00467

[17] Bjerkaas, M.L. and Riedel, F.W. (1979) Proposed Model for the Elevation Spectrum of a Wind-Roughened Sea Surface. Technical Report APL-TG-1328-I-31, Applied Physics Lab, John Hopkins University, Baltimore, 31 p.

[18] Donelan, M.A. and Pierson, W.J.P. (1987) Radar Scattering and Equilibrium Ranges in Wind-Generated Waves with Application to Scatterometry. Journal of Geophysical Research, 92, 4971-5029. http://dx.doi.org/10.1029/JC092iC05p04971

[19] Apel, J.R. (1994) An Improved Model of the Ocean Surface Wave Vector Spectrum and Its Effect on Radar Backscatter. Journal of Geophysical Research, 99, 16269-16291. http://dx.doi.org/10.1029/94jc00846

[20] Cox, C.S. and Munk, W.H. (1954) Statistics of the Sea Surface Derived from Sun Glitter. Journal of Maritime Research, 13, 198-227.

[21] Donelan, M.A., Dobsin, F.W., Smith, S.D. and Anderson, R.J. (1985) On the Dependence of Sea Surface Roughness on Wave Development. Philosophical Transactions of the Royal Society, 315, 509-562.

[22] Phillips, O.M. (1985) Spectral and Statistical Properties of the Equlibrium Range in the Wind-Generated Gravity Waves. Journal of Fluid Mechanics, 156, 505-531. http://dx.doi.org/10.1017/S0022112085002221

[23] Zavorotny, V.U. and Voronovich, A.G. (2000) Scattering of GPS Signals from the Ocean with Wind Remote Sensing Application. IEEE Transactions on Geoscience and Remote Sensing, 38, 951-964. http://dx.doi.org/10.1109/36.841977

[24] Komjathy, A., Zavorotny, V., Axelrad, P., Born, G. and Garrison, J. (2000) GPS Signal Scattering from Sea Surface: Wind Speed Retrieval Using Experimental Data and Theoretical Model. Remote Sensing of Environment, 73, 162-174. http://dx.doi.org/10.1016/S0034-4257(00)00091-2

[25] Garrison, J.L., Komjathy, A., Zavorotny, V.U. and Katzberg, S.J. (2002) Wind-Speed Measurement Using ForwardScattered GPS Signals. IEEE Transactions on Geoscience and Remote Sensing, 40, 50-65. http://dx.doi.org/10.1109/36.981349

[26] Komjathy, A., Armatys, M., Masters, D., Axelrad, P., Zavorotny, V.U. and Katzberg, S. (2004) Retrieval of Ocean Surface Wind Speed and Wind Direction Using Reflected GPS Signals. Journal of Atmospheric and Oceanic Technology, 21, 515-526. http://dx.doi.org/10.1175/1520-0426(2004)021<0515:ROOSWS>2.0.CO;2

[27] Gleason, S., Hoodgart, S., Sun, Y., Gommenginger, C., Mackin, S., Adjrad, M. and Unwin, M. (2005) Detection and Processing of Bistatically Reflected GPS Signals from Low Earth Orbit for the Purpose of Ocean Remote Sensing. IEEE Transactions on Geoscience and Remote Sensing, 43, 1229-1241. http://dx.doi.org/10.1109/TGRS.2005.845643 